\title{
Revisitingmolecular serotyping of Streptococcus pneumoniae
}

\author{
Dhian RA Camargo ${ }^{1,4}$, Fabiano S Pais ${ }^{2,3}$, Ângela C Volpini ${ }^{2,3}$, Marluce AA Oliveira ${ }^{4}$, Roney S Coimbra ${ }^{1 *}$ \\ From X-meeting 2014 - International Conference on the Brazilian Association for Bioinformatics and \\ Computational Biology \\ Belo Horizonte, Brazil. 28-30 October 2014
}

\begin{abstract}
Background: Ninety-two Streptococcus pneumoniae serotypes have been described so far, but the pneumococcal conjugate vaccine introduced in the Brazilian basic vaccination schedule in 2010 covers only the ten most prevalent in the country. Pneumococcal serotype-shifting after massive immunization is a major concern and monitoring this phenomenon requires efficient and accessible serotyping methods. Pneumococcal serotyping based on antisera produced in animals is laborious and restricted to a few reference laboratories. Alternatively, molecular serotyping methods assess polymorphisms in the cps gene cluster, which encodes key enzymes for capsular polysaccharides synthesis in pneumococci. In one such approach, cps-RFLP, the PCR amplified cps loci are digested with an endonuclease, generating serotype-specific fingerprints on agarose gel electrophoresis.
\end{abstract}

Methods: In this work, in silico and in vitro approaches were combined to demonstrate that Xholl is the most discriminating endonuclease for cps-RFLP, and to build a database of serotype-specific fingerprints that accommodates the genetic diversity within the cps locus of 92 known pneumococci serotypes.

Results: The expected specificity of cps-RFLP using Xholl was 76\% for serotyping and 100\% for serogrouping. The database of cps-RFLP fingerprints was integrated to Molecular Serotyping Tool (MST), a previously published webbased software for molecular serotyping. In addition, 43 isolates representing 29 serotypes prevalent in the state of Minas Gerais, Brazil, from 2007 to 2013, were examined in vitro; 11 serotypes (nine serogroups) matched the respective in silico patterns calculated for reference strains. The remaining experimental patterns, despite their resemblance to their expected in silico patterns, did not reach the threshold of similarity score to be considered a match and were then added to the database.

Conclusion: The cps-RFLP method with Xholl outperformed the antisera-based and other molecular serotyping methods in regard of the expected specificity. In order to accommodate the genetic variability of the pneumococci cps loci, the database of cps-RFLP patterns will be progressively expanded to include new variant in vitro patterns. The cps-RFLP method with endonuclease Xholl coupled with MST for computer-assisted interpretation of results may represent a relevant contribution to the real time detection of changes in regional pneumococci population diversity in response to mass immunization programs.

\footnotetext{
* Correspondence: roney.s.coimbra@cpqrr.fiocruz.br

${ }^{1}$ Biosystems Informatics, Research Center René Rachou (CPqRR), Oswaldo

Cruz Foundation (FIOCRUZ), Belo Horizonte, MG, 30190-002, Brazil

Full list of author information is available at the end of the article
}

(c) 2015 Camargo et al.: licensee BioMed Central Ltd. This is an Open Access article distributed under the terms of the Creative Commons Attribution License (http://creativecommons.org/licenses/by/4.0), which permits unrestricted use, distribution, and 


\section{Background}

Streptococcus pneumoniae is a Gram-positive coccus, with more than 90 serotypes, and is one of the most important agents of pneumonia, meningitis and sepsis in children worldwide [1,2]. In Brazil, between 2004 and 2006, pneumococcal disease was responsible for 34,217 hospitalizations in the Brazilian Unified Health System $(0.1 \%$ of the total number of hospitalizations), and pneumonia represented $64.8 \%$ of this total. Pneumococci also caused $31.3 \%$ of all confirmed cases of bacterial meningitis [3].

Pneumococcal disease can be prevented by vaccination. In 2010, the 10-valent pneumococcal conjugate vaccine PCV10, covering serotypes $1,4,5,7 \mathrm{~F}, 6 \mathrm{~B}, 9 \mathrm{~V}, 14,18 \mathrm{C}$, $19 \mathrm{~F}$, and $23 \mathrm{~F}$, was introduced in the Brazilian basic vaccination schedule. It is reasonable to expect that the prevalence of pneumococcal diseases in this country will be drastically reduced within a few years. However, pneumococcal serotype-shifting after massive immunization is a major concern and monitoring this phenomenon requires efficient and accessible serotyping techniques $[4,5]$.

The Quellung reaction is the gold standard method for pneumococci serotyping. This method relies on the recognition of capsular polysaccharides (CPS) by serotype or serogroup-specific antibodies. The current serotyping scheme requires a full set of expensive antisera prepared in animals, is laborious, and error-prone due to crossreactivity between some CPS [6,7]. Due to the large number of anti-sera needed for complete serotyping of bacterial isolates, laboratories with the complete panel of anti-sera are scarce. In Brazil, the Adolf Lutz Institute is the only reference center for serotyping.

Pneumococcal CPS is generally synthesized by the Wzx/Wzy-dependent pathway. The enzymes responsible for CPS synthesis are encoded by a set of genes located at the CPS biosynthetic (cps) loci, which is flanked by conserved genes $\operatorname{dex} B$ and aliA. Exceptions are serotypes 3 and 37 that use the synthase pathway $[8,9]$.

An alternative molecular serotyping method for Shigella spp. and Escherichia coli has already been published $[10,11]$. The method named $r f b$-RFLP relies on restriction fragment length polymorphisms (RFLP) of the $r f b$ loci, responsible for the synthesis of the somatic antigen in E. coli and Shigella spp. A database with $r f b$-RFLP patterns of all known serogroups/serotypes of this genospecies has been published, and a web-based software has been developed to compare the $r f b$-RFLP patterns of clinical isolates with those in the database [12]. This technique has been successfully used for more than a decade and allowed the discovery of new putative serotypes [13-15].

We present herein a new tool for S. pneumoniae molecular serotyping based on RFLP of the PCR-amplified cps locus (cps-RFLP). This tool includes: 1) a Molecular Biology method, which produces serotype-specific fingerprints; 2) a database containing the reference fingerprints; 3 ) a software to predict the serotype of clinical samples by comparing their fingerprints with those in the reference database.

\section{Methods}

All reagents were manufactured by Sigma-Aldrich (Saint Louis, MO), except when indicated in the text.

\section{Bioinformatics analysis}

One hundred and seven sequences of cps loci representing 92 serotypes were downloaded from GenBank (www.ncbi.nlm.nih.gov/Genbank) (Table S1, Additional file 1) $[9,16-18]$. When two or more sequences where available for a given serotype, all cps loci sequences were analysed to assess the diversity within serotype.

The cps sequences were screened for internal endonuclease cleavage sites using REMAP, from European Molecular Biology Open Software Suite (EMBOSS) (http://emboss.sourceforge.net). Enzymes with four to 26 restriction sites at each cps locus were selected for further analysis. For each selected enzyme, a database was built with in silico restriction patterns for each serotype generated with RESTRICT, also from EMBOSS package. This in silico analytical pipeline was applied also to the endonuclease HinfI, which had already been proposed for molecular serotyping of a subset of pneumococcal serotypes [19]. The most discriminant enzyme was chosen using a standalone version of Molecular Serotyping Tool (MST), our previously published software for computer-assisted molecular serotyping [12]. Briefly, pairwise alignments of all cps-RFLP patterns were performed and the pairwise distances were calculated as the sum of the penalties for the edit operations required to transform one pattern to the other. The most discriminant enzyme returned the highest median value for the calculated distances between all pairs of cps-RFLP patterns and the lowest number of indistinguishable pairs.

The NEIGHBOR program of Phylogeny Inference Package (PHYLIP) (http://evolution.genetics.washington. edu/phylip.html), with Unweighted Pair Group Method with Arithmetic Mean (UPGMA) as the linkage method, was used to cluster the cps-RFLP patterns based on the distance matrices produced by MST software. The dendrograms were visualized and edited with FigTree (http://tree.bio.ed.ac.uk/software/figtree/).

\section{Bacteria isolate serotyping}

Forty-five clinical isolates of S. pneumoniae representing 31 serotypes (Table 1 ) isolated at the CentralPublic Health Laboratory (FUNED) of the state of Minas Gerais, Brazil, from 2007 to 2013 and ATCC49619 reference strain (serotype 19F) were analysed. The 31 serotypes 
Table 1. Streptococcus pneumoniae isolates tested in vitro

\begin{tabular}{|c|c|c|}
\hline Strain designation & Serotype & $\begin{array}{l}\text { cps-RFLP } \\
\text { pattern }\end{array}$ \\
\hline 295/08-LP & 1 & E_1 \\
\hline 381/11-LCR, 159/11-LCR & 3 & $-*$ \\
\hline $345 / 11-L C R$ & 4 & E_4 \\
\hline 197/11-HEM & 5 & E_5 \\
\hline $\begin{array}{l}\text { 103/11-LCR, 305/08-HEM, 262/08-LCR, 619/ } \\
\text { 08-LCR }\end{array}$ & $6 \mathrm{~A}$ & E_6A \\
\hline $143 / 11-L C R$ & $6 \mathrm{~B}$ & E_6B \\
\hline 127/10-LCR, 387/11-LCR & $6 C$ & E_6C \\
\hline 176/08-LCR, 648/10-LCR & $7 C$ & E_7C \\
\hline 149/11-LCR, 620/09-LCR & $9 \mathrm{~N}$ & E_9N \\
\hline 585/10-LCR, 511/11-LCR & $9 \mathrm{~V}$ & E_9V \\
\hline 421/10-LCR, 490/11-LCR & $10 \mathrm{~A}$ & E_10A \\
\hline 084/11-LCR & $12 \mathrm{~F}$ & $E_{-} 12 \mathrm{~F}$ \\
\hline 148/10-LCR & 13 & E_13 \\
\hline 198/11-HEM, 380/12-LCR & 14 & E_14 \\
\hline 779/09-LCR & $15 C$ & $E_{-} 15 C$ \\
\hline 156/11-LCR & $16 \mathrm{~F}$ & $E_{-} 16 \mathrm{~F}$ \\
\hline 595/11-LCR & $17 \mathrm{~F}$ & $E_{-} 17 \mathrm{~F}$ \\
\hline 586/10-LCR & $18 \mathrm{~A}$ & E_18A \\
\hline 240/11-LCR & $18 \mathrm{~B}$ & $E_{-} 18 \mathrm{~B}$ \\
\hline 120/11-LCR & $18 \mathrm{C}$ & E_18C \\
\hline 124/11-LCR, 649/11-LP & $19 A$ & E_19A \\
\hline 254/11-LCR, 24/12-LCR & $19 F$ & E_19F \\
\hline ATCC49619 & $19 F$ & E_19F' \\
\hline $509 / 12-L C R$ & $19 F$ & E_19F" \\
\hline 443/10-LCR & $22 \mathrm{~F}$ & $-*$ \\
\hline 159/09-LCR & $23 B$ & E_23B \\
\hline 029/11-LCR & $23 \mathrm{~F}$ & E_23F \\
\hline 080/11-LCR & $24 \mathrm{~F}$ & E_24F \\
\hline 435/09-LCR & $28 \mathrm{~A}$ & E_28A \\
\hline 79/11-HEM & 29 & E_29 \\
\hline 317/08-LCR & 34 & E_34 \\
\hline 144/09-LCR, 192/11-LCR & $35 B$ & E_35B \\
\hline 169/11-HEM & $35 \mathrm{~F}$ & E_35F \\
\hline
\end{tabular}

* PCR amplification of cps loci failed for these isolates.

correspond to $83.7 \%$ of prevalent pneumococcal serotypes in children younger than five years in Brazil [20].

Pneumococci identification was confirmed by Gram stain, colonial morphology on blood agar, susceptibility to optochin (5 micrograms disks), and bile solubility [7]. All isolates were serotyped by Quellung reaction $[7,21]$ at Adolfo Lutz Institute, Brazilian Reference Laboratory for Bacterial Meningitis, using specific antisera (Statens Serum Institut, Copenhagen, Denmark).

\section{Genomic DNA preparation}

S. pneumoniae strains were grown overnight on blood agar and the DNA was extracted using the method originally described by Coimbra et al [10] and adapted to S. pneumoniae. Briefly, colonies were harvested from agar and resuspended in sterile saline. Cell titer was estimated by measuring the optical density in turbidimeter (Biomèrieux, Marcy l'Etoile, France). A volume containing approximately $1.8 \times 10^{8}$ bacteria was centrifuged at $3000 \mathrm{~g}$ for 15 minutes $(\mathrm{min})$ at $4^{\circ} \mathrm{C}$ and pellets were resuspended in $10 \mathrm{ml}$ of washing buffer $(1 \mathrm{M} \mathrm{NaCl}$; $10 \mathrm{mM}$ Tris- $\mathrm{HCl} ; \mathrm{pH}$ 7.6). After a second centrifugation step, the pellet was resuspended in $50 \mu \mathrm{l}$ of washing buffer, $15 \mu \mathrm{l}$ of lysozyme at $20 \mathrm{mg} / \mathrm{ml}$ and $3 \mu \mathrm{l}$ of the mutanolysin at $5 \mathrm{U} / \mathrm{ml}$. Sixty-eight microliters of $2 \%$ lowmelting-point agarose prepared with TE buffer $(10 \mathrm{mM}$ Tris-HCl; 1 mM EDTA; pH 8.0) was added to the mix. The mixture was homogenized and incubated at $41^{\circ} \mathrm{C}$ for $10 \mathrm{~min}$. Then, aliquots of $20 \mu \mathrm{l}$ were pippeted onto glass slides covered with Parafilm and let to solidify. Plugs were then transferred into $15 \mathrm{ml}$ Falcon tubes (Bacton Dickinson Labware, Franklin Lakes, NJ) containing $1 \mathrm{ml}$ of lysis buffer ( $1 \mathrm{M} \mathrm{NaCl} ; 100 \mathrm{mM}$ EDTA; $6 \mathrm{mM}$ Tris$\mathrm{HCl} ; 0.25 \%$ Brij 58 ; $0.2 \%$ deoxycholate; $0.5 \% N$-lauroylsarcosine, [pH 8.0]) supplemented with $5 \mu \mathrm{l}$ of mutanolysin $(5 \mathrm{U} / \mathrm{ml}), 50 \mu \mathrm{l}$ of lysozyme $(20 \mathrm{mg} / \mathrm{ml})$ and $10 \mu \mathrm{l}$ of RNase I $(50 \mathrm{mg} / \mathrm{ml})$ and tubes were incubated at $37^{\circ} \mathrm{C}$ overnight. After that, the lysis buffer was discarded, $1 \mathrm{ml}$ of ES buffer (0.5 M EDTA, pH 9,1\% N-lauryl sarcosyl) containing $0.1 \mathrm{mg} / \mathrm{ml}$ of proteinase $\mathrm{K}$ was added, and tubes were incubated at $51^{\circ} \mathrm{C}$ overnight. Then, ES buffer was discarded and plugs were washed six times in $10 \mathrm{ml}$ of $1 \mathrm{X}$ TE for $60 \mathrm{~min}$ at room temperature. One plug (approximately $20 \mu \mathrm{l}$ ) was melted at $68^{\circ} \mathrm{C}$ for $15 \mathrm{~min}$ in $20 \mu \mathrm{l}$ of $1 \mathrm{X}$ TE and $3 \mu \mathrm{l}$ were used as DNA templates for the PCR reaction. The DNA was evaluated for quality and quantity by electrophoresis in $0.6 \%$ agarose gels, with TBE buffer ( $89 \mathrm{mM}$ Tris-base; $89 \mathrm{mM}$ boric acid; $2.5 \mathrm{mM}$ EDTA; $\mathrm{pH} 8.0$ ), at $4.5 \mathrm{~V} / \mathrm{cm}$ between electrodes for $90 \mathrm{~min}$. The fragments sizes were roughly estimated using the lambda Hind III ladder (Promega, Madison, WI) and the GelAnalyzer software (www.gelanalyzer. com). This extraction method was chosen because it yields large and high-integrity DNA fragments suitable for long-distance PCR [10,11].

\section{PCR $c p s$ amplification}

Oligonucleotides DexB2 (5'-GAC CGT CGC TTC CTA GTT GT-3') and AliA2 (5'-ATG CAG CTA AAG TAG TCG CC-3'), respectively complementary to $\operatorname{dex} B$ and aliA [19], were used to amplify the cps gene clusters. Amplification was performed using AccuTaq LA DNA polymerase. Three microliters of template DNA was added to the amplification solution containing $0.5 \mu \mathrm{l}$ Taq (2.5 U), $5 \mu \mathrm{l}$ buffer, $1 \mu \mathrm{l}$ DMSO, deoxynucleoside triphosphates at $0.5 \mathrm{mM}$, and $0.6 \mathrm{mM}$ of primers in a final volume of $50 \mu \mathrm{l}$. Cycling conditions were programmed as follows: one denaturation step at $93^{\circ} \mathrm{C}$ for 2 min and 10 initial cycles of $93^{\circ} \mathrm{C}$ for 15 seconds (sec), 
$50^{\circ} \mathrm{C}$ for $30 \mathrm{sec}$, and $68^{\circ} \mathrm{C}$ for $20 \mathrm{~min}$, followed by 25 iterative cycles of $93^{\circ} \mathrm{C}$ for $15 \mathrm{sec}, 50^{\circ} \mathrm{C}$ for $30 \mathrm{sec}$, and $68^{\circ} \mathrm{C}$ for $20 \mathrm{~min}$ plus $15 \mathrm{sec}$ for each new cycle. A final elongation step of $68^{\circ} \mathrm{C}$ for $10 \mathrm{~min}$ was run. Amplicons were verified by electrophoresis as above. PCR product sizes were estimated using the lambda Hind III DNA ladder (Promega, Madison, WI) and the GelAnalyzer software.

\section{cps-RFLP}

Twenty-five microliters of each amplified product were digested using XhoII as follows. PCR products were incubated with $1 \mu \mathrm{l}$ enzyme (10 U), $5 \mu$ l digestion buffer (Promega, Madison, WI) for 4 hours (h) at $37^{\circ} \mathrm{C}$ according to the manufacturer's instructions. Restriction fragments were separated by electrophoresis in $20 \times 25 \mathrm{~cm}$ gels made of $1.5 \%$ ultrapure agarose (Invitrogen, Carlsbad, CA) in $0.5 \mathrm{X}$ TAE buffer (20 mM Tris-acetate; 0.5 mM EDTA; pH 8.0) at $4.5 \mathrm{~V} / \mathrm{cm}$ between electrodes for $4 \mathrm{~h}$. Standard molecular weight GeneRuler $1 \mathrm{~kb}$ Plus DNA Ladder (Thermo Scientific, Wilmington, DE) was selected as molecular marker. After electrophoresis, gels were stained for 45 min with $0.5 \mu \mathrm{g} / \mathrm{ml}$ ethidium bromide and destained twice for $15 \mathrm{~min}$ in distilled water. Gel images were electronically captured using a charge-coupled device (CCD) video camera interfaced to a microcomputer. Tagged image file format (TIFF) images were collected and the molecular weights of fragments were estimated using the GelAnalyzer, with the following parameters: Rolling ball: $25, \mathrm{MW}$ calibration: $\log$ fit. Bands corresponding to fragments smaller than 250 and larger than 4,300 bp were not considered because fragments sizing above and below these thresholds are more error-prone $[10,11]$.

\section{Reference database}

The cps-RFLP patterns obtained in silico were uploaded to the database of the web-based MST software [12], which is freely accessible at www.cebio.org/mst. In addition, the database was complemented with the in vitro patterns that did not match any of the in silico ones.

\section{Statistical analysis}

The ensembles of MST distances between all pairs of cps-RFLP patterns predicted in silico for BslFI, Eco57MI, HindII, HinfI, and StyI were compared to the ensemble predicted for XhoII using Friedman test followed by Dunn's Multiple Comparison Test. Differences were considered significant when $\mathrm{p}<0.05$. Statistical analyses were performed using GraphPad Prism 5.02 (GraphPad Software Inc, San Diego, CA).

\section{Results}

\section{Bioinformatics analysis}

In silico restriction analysis disclosed five candidate endonucleases. For each of these enzymes, namely
BslFI, Eco57MI, HindII, StyI and XhoII, a database was built with the restriction patterns calculated for each serotype. These cps-RFLP patterns were represented by strings of comma-separated, size-ordered fragments (within the thresholds from 250 to $4,300 \mathrm{bp}$ ). For each endonuclease, MST aligned all pairs of cpsRFLP patterns producing a distance matrix from which the median, mean, standard deviation and the number of indistinguishable serotype pairs under the selected threshold of 3.0 for MST distance were calculated. XhoII was the most discriminating endonuclease with the highest median distance between pairs of $c p s$-RFLP patterns and the lowest number of indistinguishable serotype pairs (Table 2). The cps-RFLP patterns predicted for XhoII consist of three to 17 restriction fragments ranging from 254 to 4,274 bp (Figure 1). MST clearly distinguished 70 in silico serotype-specific cpsRFLP patterns obtained with XhoII. However, the following pairs of serotypes were indistinguishable: 7A/7F; 9A/9V; 9L/9N; 12B/12F; 15B/15C; 18B/18C; 22A/22F; $25 \mathrm{~A} / 25 \mathrm{~F} ; 28 \mathrm{~A} / 28 \mathrm{~F} ; 32 \mathrm{~A} / 32 \mathrm{~F}$ and $33 \mathrm{~A} / 33 \mathrm{~F}$. Thus, the expected specificity was $76 \%$ for serotyping and $100 \%$ for serogrouping. For HinfI, the in silico cps-RFLP patterns had nine to 44 restriction fragments ranging from 250 to $4,120 \mathrm{bp}$, and the MST did not differentiate 235 pairs of serotypes (Table 2), resulting in an expected specificity of $15.2 \%$ for serotyping and $23.9 \%$ for serogrouping. These results confirmed XhoII as the enzyme of choice for further analysis.

\section{Experimental $c p s$ RFLP with Xholl}

PCR amplicons with 17 to $26 \mathrm{kbp}$ were obtained for 43 pneumococci isolates. After digestion with XhoII, 31 distinct patterns were obtained for 29 serotypes represented in this strains collection (the 19F strain produced three different patterns). As expected, the in vitro cps-RFLP patterns had four to 17 fragments ranging from 290 to 4,273 bp (Figure 2). To our great dismay, we did not succeed to PCR amplify the cps regions of two isolates of serotype 3 and one of serotype $22 \mathrm{~F}$ after various attempts. This is surprising since Batt and cols. [19] successfully amplified the cps region of the reference strains of these two serotypes using the same primers.

Reproducibility of the cps-RFLP patterns was confirmed in triplicate assays of 12 isolates of different serotypes randomly chosen. The maximum inter-gel variation in band sizing was $4.33 \%$ to the lower size range $(0.25-0.5 \mathrm{kbp})$ and $2.23 \%$ to the upper size range $(0.5-4.3 \mathrm{kbp})$. The intra-gel variation was $1.99 \%$ to the lower size range and $1.74 \%$ to the upper size range. These limits were lower than the default values of MST, which correspond to the maximal error tolerated in band sizing varying linearly from $7.0 \%$ at 
Table 2. Statistical analyses for identification of the most discriminating endonuclease for molecular serotyping

\begin{tabular}{lcccccc}
\hline Enzyme & $\begin{array}{c}\text { Number of } \\
\text { indistinguishable } \\
\text { pairs of serotypes }\end{array}$ & $\begin{array}{c}\text { Specificity } \\
\text { for } \\
\text { serotyping }\end{array}$ & $\begin{array}{c}\text { Specificity } \\
\text { for } \\
\text { serogrouping }\end{array}$ & $\begin{array}{c}\text { Median pairwise } \\
\text { distances between } \\
\text { cps-RFLP patterns }\end{array}$ & $\begin{array}{c}\text { Mean pairwise distance } \\
\text { between cps-RFLP patterns } \pm \\
\text { Standard Deviation }\end{array}$ & $\begin{array}{c}\text { Median significantly } \\
\text { different when } \\
\text { compared to Xholl } \\
\text { (p < 0.05) }\end{array}$ \\
\hline BsIFl & 17 & $66.3 \%$ & $85.8 \%$ & 29.6 & $31.6 \pm 15.7$ & Yes \\
Eco57Ml & 23 & $56.5 \%$ & $89.1 \%$ & 30.5 & $32.0 \pm 15.2$ & Yes \\
Hindll & 27 & $54.3 \%$ & $75 \%$ & 29.8 & $31.8 \pm 16.0$ & Yes \\
Hinfl & 235 & $15.2 \%$ & $23.9 \%$ & 8.6 & $10.0 \pm 6.4$ & Yes \\
Styl & 24 & $63 \%$ & $76 \%$ & 32.1 & $34.6 \pm 18.4$ & No \\
Xholl & 11 & $76 \%$ & $100 \%$ & 32 & $34.5 \pm 17.8$ & \\
\hline
\end{tabular}

$0.5 \mathrm{kbp}$ to $3.5 \%$ at $4 \mathrm{kbp}$. Thus, the default parameterization of the MST was maintained.

\section{Comparison of the in vitro and in silico cps-RFLP patterns}

Forty-two clinical isolates of 29 serotypes yielded in vitro cps-RFLP patterns, and 11 of these serotypes matched their respective in silico patterns. The vast majority of pairs of in silico and in vitro patterns of the same serotype were similar (Figure 3), as expected, even when the score of the MST alignment was greater than the threshold of 3.0 and the alignments were not considered to be a match. Only eight pairs of patterns were markedly unrelated (Figure 4).

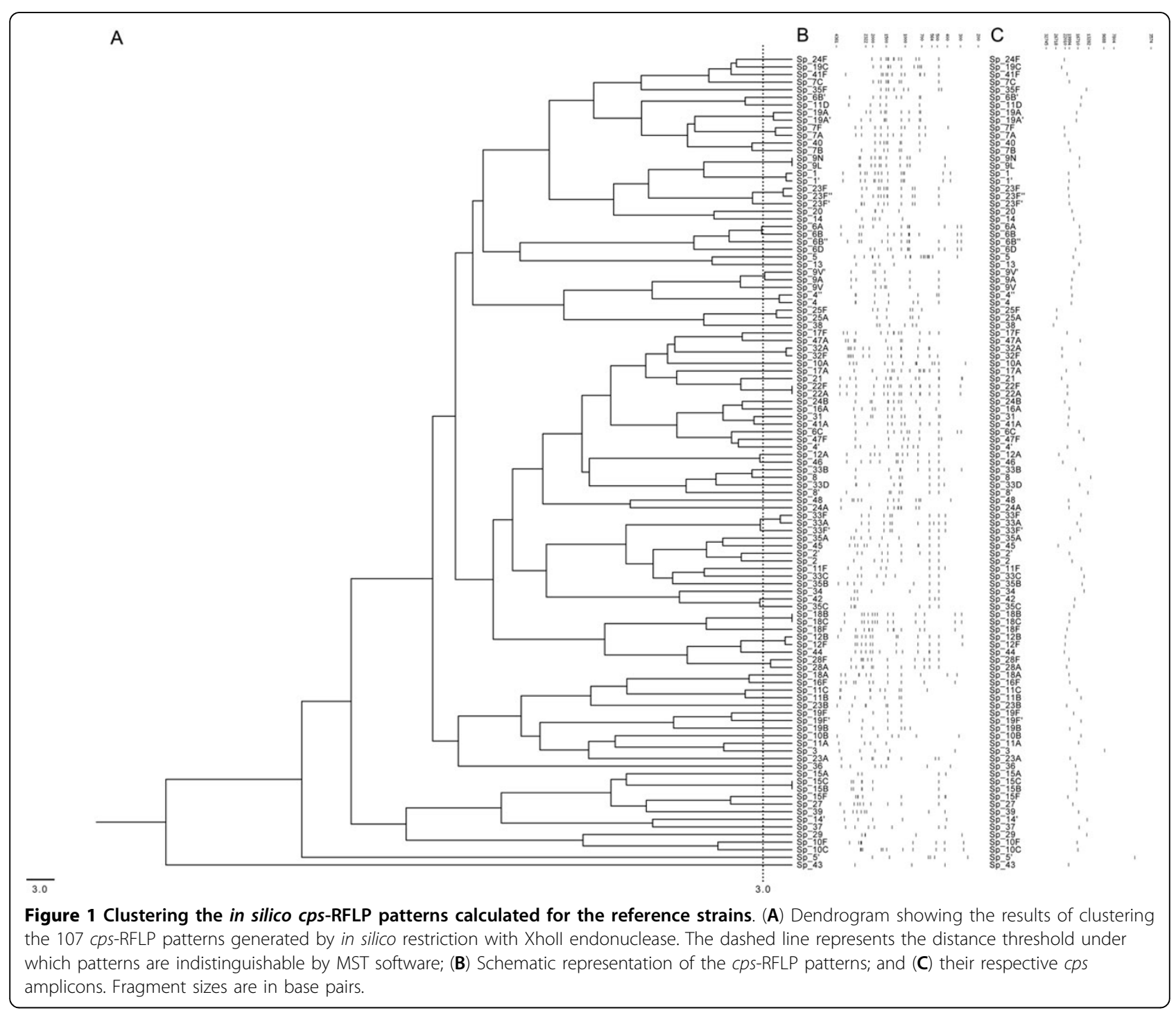




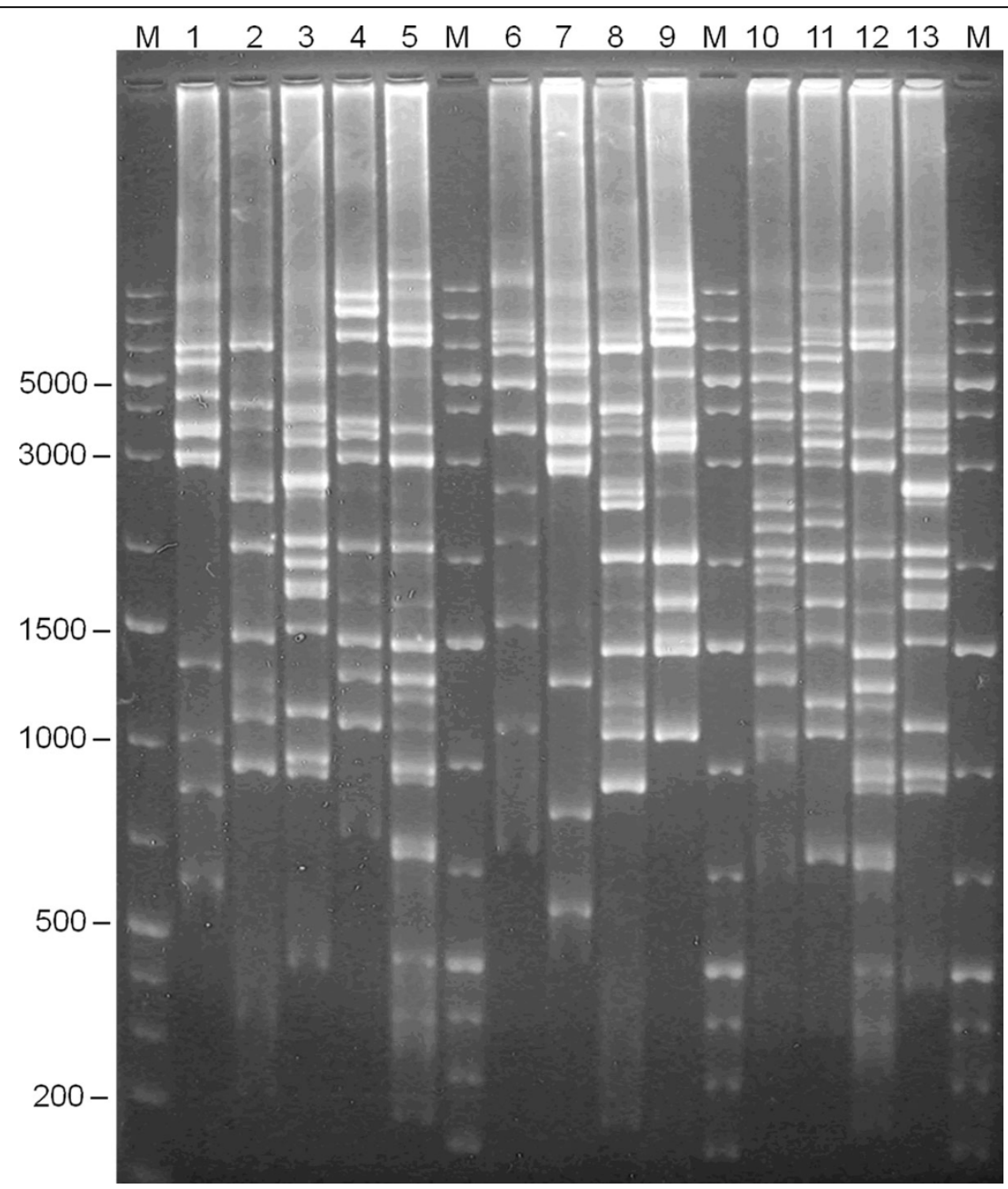

Figure 2 cps-RFLP experimental patterns of serotypes 29, 9N, 19F, 6C, 19A, 29, 6A, 19F, 18B, 6B. Experimental cps-RFLP patterns in agarose gel (1.5\%). Lanes: $M=$ molecular weight marker; 1 = 79/11-HEM (serotype 29); 2 = 103/11-LCR (serotype 6A); $3=149 / 11-L C R$ (serotype 9N); 4 = 24/12-LCR (serotype 19F); 5 = 387/11-LCR (serotype 6C); 6 = 124/11-LCR (serotype 19A); 7 = 79/11-HEM (serotype 29); $8=103 / 11-L C R$ (serotype 6A); 9 = ATCC49619 (serotype 19F); 10 = 240/11-LCR (serotype 18B); $11=143 / 11-L C R$ (serotype 6B); 12 = 387/11-LCR (serotype 6C); $13=149 / 11-L C R$ (serotype 9N). Fragment sizes are in base pairs.

The MST reference database was loaded with the 107 in silico cps-RFLP patterns and with 19 in vitro patterns that did not match any previous in silico pattern under the threshold of similarity score. Altogether, these cpsRFLP patterns represent 92 known pneumococci serotypes.

\section{Discussion}

The molecular serotyping method presented herein, cpsRFLP with endonuclease XhoII, assesses polymorphisms in the cps region of Streptococcus pneumoniae allowing serotype identification. In this work, we combined in silico and in vitro approaches to produce a database of serotypespecific cps-RFLP fingerprints that accommodates the genetic diversity within the cps locus of 92 known pneumococci serotypes. The database was integrated to MST producing the largest freely accessible dataset of restriction patterns of the cps loci of $S$. pneumoniae isolates from different geographical origins.

The Quellung reaction [7,21]with antisera is still the gold-standard for pneumococcal serotyping. However, sera cross-reactivity have already been reported to at least 13 serotype pairs $(1 / 7 \mathrm{C}, 7 \mathrm{~A} / 7 \mathrm{C}, 7 \mathrm{C} / 7 \mathrm{~F}, 9 \mathrm{~A} / 9 \mathrm{~V}, 9 \mathrm{~N} /$ $9 \mathrm{~V}, 9 \mathrm{~L} / 9 \mathrm{~N}, 10 \mathrm{~A} / 10 \mathrm{~B}, 10 \mathrm{~A} / 33,18 \mathrm{~B} / 18 \mathrm{C}, 18 \mathrm{~B} / 18 \mathrm{~F}, 19 \mathrm{~A} /$ $19 \mathrm{~F}, 42 / 35 \mathrm{C}$ and 48/45) [22]. From these, only the pairs $9 \mathrm{~A} / 9 \mathrm{~V}, 9 \mathrm{~L} / 9 \mathrm{~N}$ and $18 \mathrm{~B} / 18 \mathrm{C}$, could not be distinguished by cps-RFLP with Xholl during the in silico analysis. They belong to the same serogroups and have very similar cps sequences [20].

The cps-RFLP patterns were obtained, in vitro, using the endonuclease XhoII, for a set of clinical isolates of a welldefined geographical region representing 29 serotypes. 


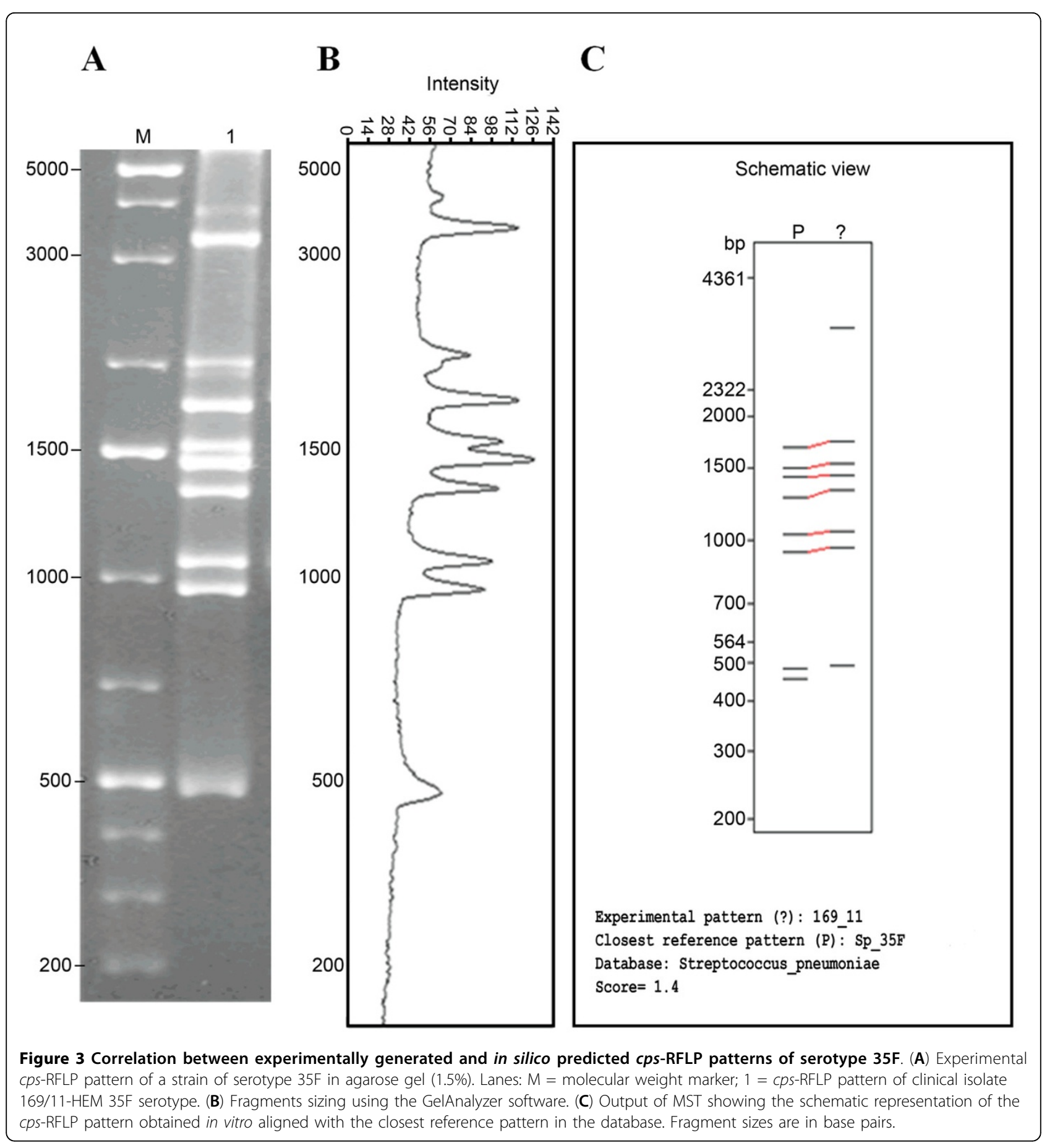

Eleven of these serotypes matched their respective predicted patterns in the in silico database (37.9\%). The large majority of the cps-RFLP patterns obtained in vitro and their correspondent patterns predicted in silico were highly similar (Figure 4). The slight differences observed might be in part explained by the fact that the primers used in the present work [19] are different from those used by Bentley and cols. [9] who sequenced most of the pneumococcal cps regions available in Genbank. In fact, we have failed to amplify the $c p s$ locus using the primers published by Bentley and cols. as reported by others [23]. Some minor differences can also be explained by incomplete digestion, inaccurate fragments sizing, or by comigration of fragments with very similar molecular weights. The problem of incomplete digestion can be minimized by using an internal control in each experiment 


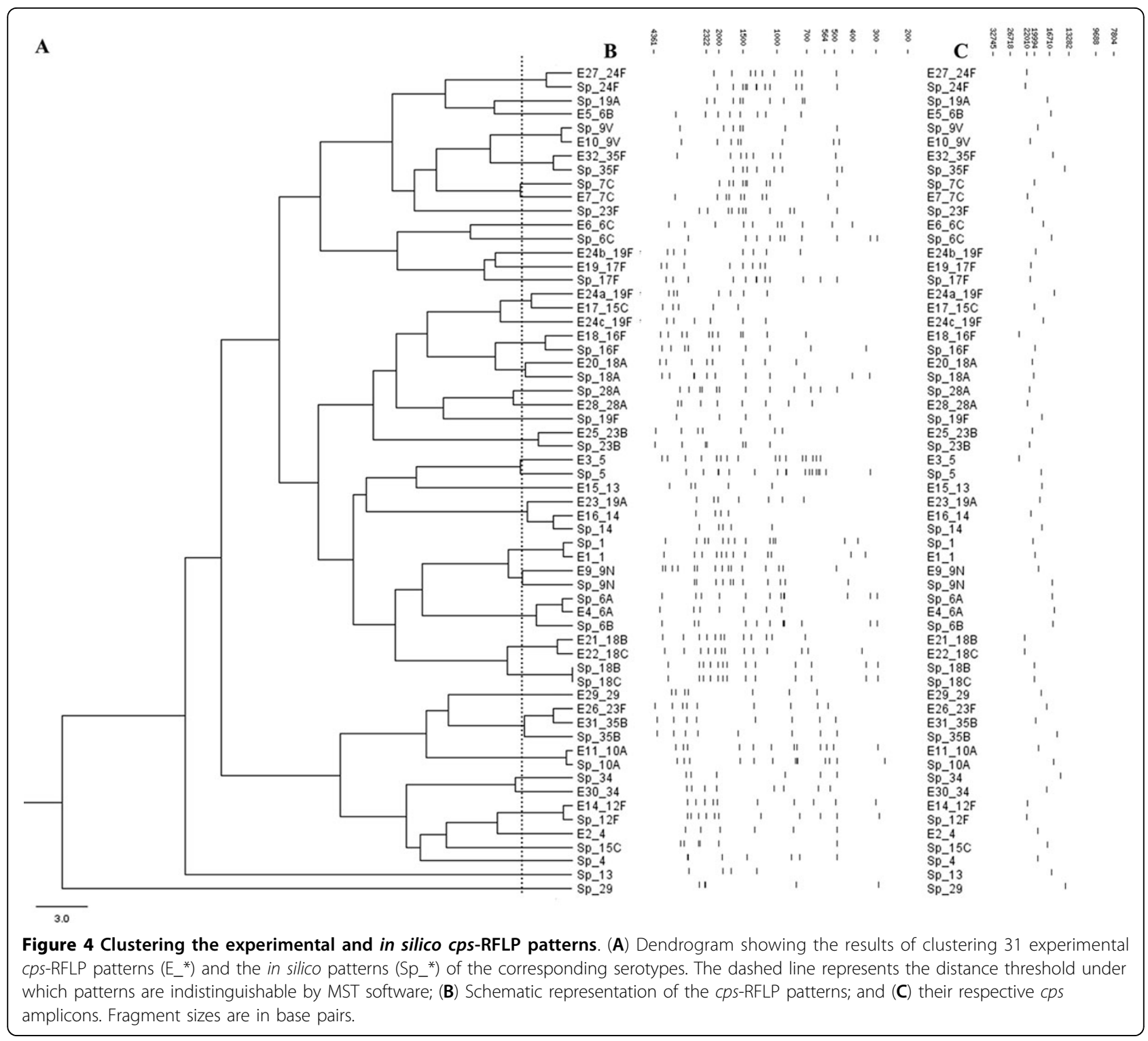

(a strain with previously stablished cps-RFLP pattern). However, it is worth noting that, in our reproducibility assays, described in the Results section, all cps-RFLP patterns obtained for a same strain in different assays were highly similar, and the slight intra- and inter-gel variations could easily be handled by the algorithm of MST. Finally, it is possible that variant patterns for the same serotype may reflect real polymorphisms caused by silent point mutations, insertions, or deletions in the cps region that do not alter the CPS antigenic structure. Three different strains of serotype 19F produced three different cps-RFLP patterns in vitro, most probably due to silent polymorphisms in the XhoII sites in the cps region that did not alter the CPS antigenic structure. Similar results had been previously reported in S. pneumoniae [16,19], Shigella [10], and E. coli [11]. Therefore, in order to accommodate this variability, the database of $c p s$-RFLP patterns with XhoII was complemented with in vitro patterns that did not match those predicted in silico.

Molecular serotyping methods based on polymorphisms of the cps region of $S$. pneumoniae have been proposed before. Batt and cols. [19] used HinfI to digest the PCR-amplified cps regions of 81 epidemiologically unrelated strains representing 46 different serotypes. The patterns obtained were loaded to a database. Afterwards, those authors tested their method against an independent set of 73 isolates from their regional collection, and 43 matched patterns in the database (58.9\%). However, it is worth to note that the observed specificity of serotyping methods may be biased by the fact that any single strain collection is differently enriched by serotypes circulating in the geographic regions where 
samples were collected. Accordingly, our in silico simulation demonstrated that cps-RFLP with HinfI would have only $15.2 \%$ specificity when the 92 known serotypes are considered (Table 2). Additionally, all serotype pairs that were indistinguishable with Xholl could not be differentiated with HinfI. Therefore, the use of endonuclease XhoII significantly increased the specificity of cps-RFLP method.

Molecular serotyping methods based on multiplex PCR and microarrays have also been proposed. Yun and cols. [24] developed a multiplex PCR assay to cover the pneumococcal serotypes prevalent in Korea, and Jourdain and cols. [25] designed another version targeting pneumococci epidemiologically relevant in Belgium. These methods require up to eight sequential PCR reactions and amplicon detection steps, and are not readily portable to other geographic regions where pneumococci have different population structures. Alternatively, Raymond et al. [26] described a microarray-based assay to identify S. pneumoniae serotypes or serogroups. Assessing 12 polymorphisms located in the capsular operon these authors identified 22 serotypes and assigned 24 other serotypes to a subgroup of serotypes. Another research group developed a microarray incorporating oligonucleotide probes for all known capsular polysaccharide synthesis genes. This array failed to identify only two serotypes in a panel of 91 reference strains representing 91 serotypes [27]. However, further studies with clinical strains from different geographic regions, which can have polymorphisms that are not represented in the microarray, are needed to evaluate the portability of this method. Although promising, microarrays-based molecular serotyping is expensive, and requires statistical analysis of the array intensity data, rendering it unsuitable to be used by researchers in the field of $S$. pneumoniae who are unfamiliar with statistics and bioinformatics. Contrarily, cps-RFLP circumvents the main drawbacks of multiplex PCR and microarraysbased techniques, while still achieving $76 \%$ of expected specificity for serotyping and $100 \%$ for serogrouping. Moreover, cps-RFLP performed well in a panel of clinical samples representative of the pneumococci population prevalent in Brazil. Specific PCR assays can be developed with primers designed to detect genes, or gene regions specific of the few serotypes unidentified by cps-RFLP. Finally, it is worth note that cps-RFLP allows the detection of new serotypes, whose cps-RFLP patterns can be added to the database of MST.

The cps-RFLP with XhoII could be further improved with a faster DNA purification step. We have also tested the Wizard Genomic DNA Purification Kit (Promega) for long genomic DNA extraction. However, contrarily to previous reports $[19,28]$, the DNA fragments were often degraded and unsuitable for long-extension PCR amplification (data not shown). We have also compared gel electrophoresis and Agilent 2100 Bioanalyzer system (Agilent Technologies, Palo Alto, CA) for separation of fragments in the informative size range of 250 to $4300 \mathrm{bp}$. For this purpose we used the Agilent DNA $7500 \mathrm{Kit}$, which covers the size range from 100 to $7500 \mathrm{bp}$. Bioanalyzer did not perform well with this kit in the size range between 2000 and $4300 \mathrm{bp}$, where most fragments are concentrated in the cps-RFLP patterns (data not shown). Aligned with the main purpose of the present work, which is to provide regional reference laboratories with a simple, low cost, and reliable molecular serotyping method, we cannot recommend Bioanalyzer for the separation of restriction fragments in cps-RFLP method until a new kit designed to work in the informative range of fragments sizes is available. Finally, in order to accommodate the genetic variability of the pneumococci $c p s$ loci, the database of $c p s$-RFLP patterns will be progressively expanded to include new variant in vitro patterns whenever necessary.

\section{Conclusions}

The cps-RFLP method with XhoII as endonuclease and MST for computer-assisted identification of patterns obtained in vitro clearly distinguished the large majority of known pneumococcal serotypes. It thus represents a suitable alternative to the Quellung reaction, particularly for small local laboratories that usually only collect the bacterial isolates and send them to reference laboratories to be serotyped. Another advantage of cps-RFLP, when compared to other molecular serotyping techniques, is to allow the identification of the capsular ancestry of isolates rendered nonencapsulated due to Single Nucleotide Polimorphisms [29], or even larger indels. When the distance threshold is set to 3.0, the algorithm of MST can handle small variations in fragments sizes and even missing or unexpected fragments in the cps-RFLP patterns. The complete database of $c p s$-RFLP patterns obtained with XhoII is freely accessible via MST website (www.cebio.org/mst), allowing surveillance of local pneumococcal diversity by researchers from any laboratory minimally equipped for Molecular Biology. This may represent a relevant contribution to real time detection of changes in regional pneumococcal population structure in response to recently introduced mass immunization programs.

\section{Additional material}

Additional file 1: This table contains the Genbank accession numbers of the cps sequences used in this work.

List of abbreviations

CPS - Pneumococcal capsular polysaccharide; MST - Molecular Serotyping Tool; PCV10 - 10-valent pneumococcal conjugate vaccine; RFLP - Restriction 
fragment length polymorphism; UPGMA - Unweighted Pair Group Method with Arithmetic Mean.

\section{Competing interests}

The authors declare that they have no competing interests.

\section{Authors' contributions}

DRAC carried out the microbiological analysis, contributed to the statistical and bioinformatical analysis, and drafted the manuscript.

FSP contributed to the bioinformatical analysis, and drafted the manuscript ACV contributed to data analysis, and drafted the manuscript.

MAAO contributed to the microbiological analysis and drafted the manuscript.

RSC conceived and coordinated this study, contributed to the bioinformatical, statistical and microbiological analysis, and wrote the manuscript.

All authors read and approved the final manuscript before submission.

\section{Acknowledgements}

The authors thank the Program for Technological Development in Tools for Health PDTIS FIOCRUZ (RPT04B - Bioinformatics) for use of its facilities, and Francislon S. Oliveira and Michelle L. Samuel for technical support. FAPEMIG (PPM-00614-11), and CNPq (479543/2012-7) funded this work.

\section{Declarations}

Costs for article publication came from FIOCRUZ.

This article has been published as part of BMC Genomics Volume 16 Supplement 5, 2015: Proceedings of the 10th International Conference of the Brazilian Association for Bioinformatics and Computational Biology ( $X$ Meeting 2014). The full contents of the supplement are available online at http://www.biomedcentral.com/bmcgenomics/supplements/16/S5.

\section{Authors' details}

'Biosystems Informatics, Research Center René Rachou (CPqRR), Oswaldo Cruz Foundation (FIOCRUZ), Belo Horizonte, MG, 30190-002, Brazil. ${ }^{2}$ Genomics and Computational Biology Group, CPqRR, FIOCRUZ, Belo Horizonte, MG, 30190-002, Brazil. ${ }^{3}$ Center for Excellence in Bioinformatics, CPqRR, FIOCRUZ, Belo Horizonte, MG, 30190-002, Brazil. ${ }^{4}$ Service of Bacterial and Fungal Diseases, Ezequiel Dias Foundation (FUNED), Belo Horizonte, MG, 30510-010, Brazil.

Published: 26 May 2015

\section{References}

1. O'Brien KL, Wolfson LJ, Watt JP, Henkle E, Deloria-Knoll M, McCall N, Lee E, Mulholland K, Levine OS, Cherian T, et al: Burden of disease caused by Streptococcus pneumoniae in children younger than 5 years: global estimates. Lancet 2009, 374(9693):893-902.

2. Bratcher $\mathrm{PE}, \mathrm{Kim} \mathrm{KH}$, Kang JH, Hong JY, Nahm MH: Identification of natural pneumococcal isolates expressing serotype $6 \mathrm{D}$ by genetic, biochemical and serological characterization. Microbiology 2010, 156(Pt 2):555-560.

3. Novaes HM, Sartori AM, Soárez PC: Hospitalization rates for pneumococcal disease in Brazil, 2004 - 2006. Rev Saude Publica 2011, 45(3):539-547.

4. Pichon B, Ladhani SN, Slack MP, Segonds-Pichon A, Andrews NJ, Waight PA, Miller E, George R: Changes in molecular epidemiology of streptococcus pneumoniae causing meningitis following introduction of pneumococcal conjugate vaccination in England and Wales. J Clin Microbiol 2013, 51(3):820-827.

5. Rudolph K, Bruce MG, Bulkow L, Zulz T, Reasonover A, Harker-Jones M, Hurlburt D, Hennessy TW: Molecular epidemiology of serotype 19A Streptococcus pneumoniae among invasive isolates from Alaska, 19862010. Int J Circumpolar Health 2013, 72

6. Henrichsen J: Six newly recognized types of Streptococcus pneumoniae. J Clin Microbiol 1995, 33(10):2759-2762.

7. Perilla MJ, Ajello G, Bopp C, Elliot J, Facklam R, Knapp JS, Popovic T, Wells J, Dowell SF: Manual for Identification and Antimicrobial Susceptibility Testing of Bacterial Pathogens of Public Health Importance in the Developing World. Geneva: World Health Organization; 2003.

8. Jiang SM, Wang $L$, Reeves PR: Molecular characterization of Streptococcus pneumoniae type $4,6 \mathrm{~B}, 8$, and $18 \mathrm{C}$ capsular polysaccharide gene clusters. Infect Immun 2001, 69(3):1244-1255.
9. Bentley S, Aanensen D, Mavroidi A, Saunders D, Rabbinowitsch E, Collins M, Donohoe K, Harris D, Murphy L, Quail M, et al: Genetic analysis of the capsular biosynthetic locus from all 90 pneumococcal serotypes. PLoS Genet 2006, 2(3):e31.

10. Coimbra RS, Grimont F, Grimont PA: Identification of Shigella serotypes by restriction of amplified O-antigen gene cluster. Res Microbiol 1999, 150(8):543-553.

11. Coimbra RS, Grimont $F$, Lenormand $P$, Burguière $P$, Beutin $L$, Grimont $P A$ : Identification of Escherichia coli O-serogroups by restriction of the amplified O-antigen gene cluster (rfb-RFLP). Res Microbiol 2000, 151(8):639-654.

12. Coimbra RS, Artiguenave F, Jacques LS, Oliveira GC: MST (molecular serotyping tool): a program for computer-assisted molecular identification of Escherichia coli and Shigella O antigens. J Clin Microbiol 2010, 48(5):1921-1923.

13. Coimbra RS, Lenormand P, Grimont F, Bouvet P, Matsushita S, Grimont PA Molecular and phenotypic characterization of potentially new Shigella dysenteriae serotype. J Clin Microbiol 2001, 39(2):618-621.

14. Grimont F, Lejay-Collin M, Talukder KA, Carle I, Issenhuth S, Le Roux K, Grimont PA: Identification of a group of shigella-like isolates as Shigella boydii 20. J Med Microbiol 2007, 56(Pt 6):749-754.

15. Melito PL, Woodward DL, Munro J, Walsh J, Foster R, Tilley P, Paccagnella A, Isaac-Renton J, Ismail J, Ng LK: A novel Shigella dysenteriae serovar isolated in Canada. J Clin Microbiol 2005, 43(2):740-744.

16. Mavroidi A, Aanensen DM, Godoy D, Skovsted IC, Kaltoft MS, Reeves PR, Bentley SD, Spratt BG: Genetic relatedness of the Streptococcus pneumoniae capsular biosynthetic loci. J Bacteriol 2007, 189(21):7841-7855.

17. Park $\mid H$, Park S, Hollingshead SK, Nahm MH: Genetic basis for the new pneumococcal serotype, 6C. Infect Immun 2007, 75(9):4482-4489.

18. Bratcher PE, Park $I H$, Oliver MB, Hortal M, Camilli R, Hollingshead SK, Camou T, Nahm MH: Evolution of the capsular gene locus of Streptococcus pneumoniae serogroup 6. Microbiology 2011, 157(Pt 1):189-198.

19. Batt SL, Charalambous BM, McHugh TD, Martin S, Gillespie SH: Novel PCRrestriction fragment length polymorphism method for determining serotypes or serogroups of Streptococcus pneumoniae isolates. J Clin Microbiol 2005, 43(6):2656-2661.

20. Gabastou J-M: Informe Regional SIREVA II 2012: Datos por país y por grupos de edad sobre las características de los aislamientos de Streptococcus pneumoniae, Haemophilus influenzae y Neisseria meningitidis, en procesos invasores. Washington: Organización Panamericana de la Salud 2013

21. Sørensen UB: Typing of pneumococci by using 12 pooled antisera. J Clin Microbiol 1993, 31(8):2097-2100

22. Konradsen HB, Europe PRli: Validation of serotyping of Streptococcus pneumoniae in Europe. Vaccine 2005, 23(11):1368-1373.

23. Kilian M, Poulsen K, Blomqvist $T$, Håvarstein LS, Bek-Thomsen M, Tettelin H, Sørensen UB: Evolution of Streptococcus pneumoniae and its close commensal relatives. PLoS One 2008, 3(7):e2683.

24. Yun KW, Cho EY, Hong KB, Choi EH, Lee HJ: Streptococcus pneumoniae type determination by multiplex polymerase chain reaction. J Korean Med Sci 2011, 26(8):971-978.

25. Jourdain S, Drèze PA, Vandeven J, Verhaegen J, Van Melderen L, Smeesters PR: Sequential multiplex PCR assay for determining capsular serotypes of colonizing S. pneumoniae. BMC Infect Dis 2011, 11:100

26. Raymond F, Boucher N, Allary R, Robitaille L, Lefebvre B, Tremblay C, Corbeil J, Gervaix A: Serotyping of Streptococcus pneumoniae based on capsular genes polymorphisms. PLoS One 2013, 8(9):e76197.

27. Newton R, Hinds J, Wernisch L: Empirical Bayesian models for analysing molecular serotyping microarrays. BMC Bioinformatics 2011, 12:88.

28. Brisse S, Issenhuth-Jeanjean S, Grimont PA: Molecular serotyping of Klebsiella species isolates by restriction of the amplified capsular antigen gene cluster. J Clin Microbiol 2004, 42(8):3388-3398.

29. Schaffner TO, Hinds J, Gould KA, Wüthrich D, Bruggmann R, Küffer M, Mühlemann K, Hilty M, Hathaway LJ: A point mutation in cpsE renders Streptococcus pneumoniae nonencapsulated and enhances its growth, adherence and competence. BMC Microbiol 2014, 14:210.

doi:10.1186/1471-2164-16-S5-S1

Cite this article as: Camargo et al:: Revisitingmolecular serotyping of Streptococcus pneumoniae. BMC Genomics 2015 16(Suppl 5):S1. 